\title{
Modelando a Interação entre Sistema Imunológico Inato e Bactérias Estreptococos e Estafilococos ${ }^{1}$
}

YU JUN 2 , H.M. YANG 3 , Departamento de Matemática Aplicada, Instituto de Matemática, Estatística e Computação Científica, UNICAMP, Cx.P. 6065, 13083-859 Campinas, SP, Brasil e Lab-epifisma.

Resumo. Bactérias que vencem a barreira física (pele ou mucosas do trato digestivo e intestinal) provocam uma reação inflamatória localizada. Essa reação é a resposta do sistema imunológico inato para conter invasão de micro-organismos ao corpo humano. Desenvolve-se um modelo matemático para descrever essa resposta do sistema imunológico, levando em consideração a patogenicidade das bactérias. Aplicase os resultados para explicar a diferença de comportamento entre as bactérias estafilococos e estreptococos. O modelo mostra que quanto menor for a patogenicidade da bactéria, maiores são possibilidades de superar a resposta do sistema imunológico inato e causar uma infecção generalizada.

\section{Introdução}

O conjunto das células e substâncias responsáveis pela defesa do organismo, bem como suas respectivas ações, é denominado sistema imunológico (SI). O sistema imunológico dos seres mais complexos caracteriza-se por ser um sistema especializado, descentralizado e inteligente. A especialização diz respeito ao fato de que durante toda a nossa existência somos submetidos à invasão dos mais diversos tipos de patôgenos, cada um deles com sua forma específica de invasão, despistamento e reprodução. Além dos ataques externos, nosso organismo está constantemente produzindo células cancerígenas, as quais também devem ser eliminados. O SI humano deve ser capaz de responder diferentes tipos de agressões para sobrevivência.

A pele normal possui papel crítico na defesa contra uma variedade de patógenos. A interação entre patógeno e hospedeiro ainda não é totalmente elucidada e estariam envolvidos fatores bacterianos, funções de barreira da pele e fatores do hospedeiro. A infecção cutânea, freqüentemente, surge em decorrência de ruptura da integridade da epiderme. A infecção instala-se com a invasão da derme e do subcutâneo pelo patógeno e mecanismos inflamatórios são elicitados como resposta à invasão. Em geral, essa resposta imunológica localizada com reação inflamatória para isolar o local de infecção com a atuação de células como macrófagos e neutrófilos consegue proteger contra invasão de micro-organismos. Se essa primeira resposta for incapaz

\footnotetext{
${ }^{1}$ Apoio financeiro Fapesp (Projeto temático) e CNPq (Edital BIC e Edital Universal)

${ }^{2}$ Bolsista BIC (yjun@ime.unicamp.br)

${ }^{3}$ Bolsista CNPq (hyunyang@ime.unicamp.br)
} 
de conter a infecção, o SI adaptativo é ativado. Na resposta do SI adaptativo, não se observa a ação de um gerenciador central que determina as várias etapas da resposta imunológica. Pelo contrário, encontra-se efetivada em diversos pontos do organismo, como nos órgãos linfáticos periféricos estrategicamente alocados nas várias portas de entrada utilizadas pelos invasores, a fim de promover uma resposta imunológica completa e rápida contra a invasão. Muitas vezes a diferença entre a sobrevivência e a morte encontra-se na rapidez com que um invasor é debelado. A memória imunológica permite com que o SI adaptativo responda com maior rapidez a um invasor, desde que já se tenha tido contato prévio com o mesmo. Essa peculiaridade baseia-se no fato de que, uma vez devidamente exposto a um dado patôgeno, o SI tem a capacidade de guardar entre seus milhares de linfócitos (divididos em dois grupos, células B, produzidas na medula óssea, e $\mathrm{T}$, produzidas no timo) alguma adaptação direcionada àquele invasor. Temos, então, o que se convencionou denominar sistema inteligente [1].

Notamos que, por causa da sua formação, constituição, especialização e, também, da sua maneira de atuar, a resposta do SI pode ser modelada matematicamente. Os modelos matemáticos que procuram explicar algum aspecto do funcionamento do SI, ou ressaltar algum comportamento que não pode ser observado claramente em experimentos, podem trazer benefícios à própria imunologia. Além disso, tais estudos podem ser interessantes quando pensamos nas implicações em outros ramos de ciência médica, como por exemplo, a Epidemiologia que estuda os diferentes fatores que intervêm na difusão e propagação de doenças, sua freqüência, seu modo de distribuição, sua evolução e a colaboração dos meios necessários para a sua prevenção [1] [9]. Nesse trabalho desenvolve-se um modelo matemático para descrever os primeiros momentos da resposta do SI inato contra a infecção nos tecidos moles causados pelas bactérias estafilococos e estreptococos. Na seção 2 propomos e analisamos o modelo, e a conclusão é dada na secção 3.

\section{Modelagem Matemática}

As bactérias extraceulares como Staphyococcus e Streptococcus podem se multiplicar fora da célula do hospedeiro. As suas toxinas liberadas durante o seu processo de colonização levam a destruição do tecido. Os tecidos lesionados liberam várias substâncias que provocam importantes alterações secundárias no tecido. Várias destas substâncias ativam fortemente o sistema dos macrófagos. Todo o complexo dessas alterações é conhecido como inflamação.

Um dos principais resultados da inflamação é o "enclausuramento" da área de lesão em relação aos outros tecidos, retardando a propagação das bactérias e seus produtos tóxicos. A resposta dos macrófagos a inflamação é dada dentro de poucos minutos, os macrófagos que já estão presentes no tecido começam imediatamente sua ação fagocítica. O primeiro efeito da resposta consiste no rápido aumento do número dos macrófagos, tornando-os móveis dentro da primeira hora da infecção. Quando os macrófagos engolfam grandes quantidades de tecidos necróticos e bactérias, quase todos eles eventualmente morrem. Depois de vários dias, forma uma cavidade contendo quantidade variável de tecido necrótico, neutrófilos e macrófagos mortos. Esta 
cavidade é conhecida como pus. Uma vez suprimida a infecção, as células mortas e tecidos necróticos sofrem autólise e o produto resultante é absorvido pelo tecido circundante até o desaparecimento da lesão tecidual. Durante todo o processo inflamatório, observa-se que a sua intensidade costuma ser proporcional ao grau de lesão tecidual.

Desenvolvendo um modelo matemático, estuda-se a interação da primeira resposta do SI inato à inflamação provocada pelas bactérias estafilococos e estreptococos. A população das bactérias invasoras é representada por $B$, cujo crescimento é limitado pela capacidade de suporte oferecido pelo local da invasão e o posterior confinamento da sua propagação limitando a sua colonização (designada por $C$ ). Essas bactérias são fagocitadas por macrófagos, que representam todas as células do sistema imunológico. As células teciduais (designadas por $P$ ) são destruídas pelas toxinas a uma quantidade proporcional à concentração das bactérias presente no organismo do hospedeiro. Para combater as bactérias, população dos macrófagos (designada por $M$ ) migram para local de inflamação proporcionalmente à quantidade das células mortas e a um fator $f$, que pode depender ou não de $M$. O fator $f$ descreve se a migração dos macrófagos é estimulada unicamente pela quantidade de células teciduais destruídas $(f=1)$ ou é estimulada proporcionalmente pelas células destruídas e população já existente de macrófagos no local $(f=M)$. Todas as quantidades referem-se aos valores do local de infecção.

A dinâmica da interação entre SI inato e bactérias é descrita pelo seguinte sistema de equações diferenciais ordinárias:

$$
\left\{\begin{array}{l}
\frac{d B(t)}{d t}=k_{B}\left[1-\frac{B(t)}{C}\right] B(t)-\mu_{B} B(t)-\alpha B(t) M(t) \\
\frac{d P(t)}{d t}=k_{P}-\mu_{P} P(t)-\beta B(t) P(t) \\
\frac{d M(t)}{d t}=k_{M}-\left(\mu_{M}+\overline{\mu_{B}}\right) M(t)+\gamma\left[P_{0}-P(t)\right] f
\end{array}\right.
$$

onde $P_{0}$ é população inicial das células teciduais (vide próxima sub-secção). Os parâmetros $k_{B}, k_{P}$ e $k_{M}$ são as taxas de reprodução das bactérias, de células do tecido e de macrófagos, respectivamente; $\mu_{B}, \mu_{P}$ e $\mu_{M}$ são as taxas per-capitas de mortalidade natural das bactérias, de células de tecido e de macrófagos, respectivamente; $\overline{\mu_{B}}$ é a taxa de mortalidade eventual dos macrófagos após do engolfamento das bactérias e dos tecidos necróticos para a posterior formação de pus; e $\alpha, \beta$ e $\gamma$ são as taxas de destruição de bactérias por macrófagos, de morte de células teciduais e de atração dos macrófagos ao local inflamatório, respectivamente.

O modelo proposto assume homeostasia quanto a células mucosas que revestem trato gástro-intestinal ou epitelias e células da defesa. As células da defesa, genericamente denominadas macrófagos, combatem as bactérias através de fagocitose, e liberam citocinas que atraem outros macrófagos. A morte das células deve-se à ação das toxinas produzidas pelas bactérias. Por isso, assume-se que a produção de toxinas seja proporcional ao número de bactérias.

Estuda-se o sistema de equações (2.1) em estado estacionário. O modelo é analisado conforme o modo de atração de macrófagos para o local de infecção, a uma dada função $f$. 


\subsection{Macrófagos Atraídos pelas Células Mortas}

Esse sub-modelo assume que novos macrófagos são atraídos por interleucinas, que são produzidas a uma taxa de produção total constante $\gamma$ e pela quantidade de células teciduais mortas. Nesse caso o sistema dinâmico (2.1) é escrito como

$$
\left\{\begin{aligned}
\frac{d B(t)}{d t} & =k_{B}\left[1-\frac{B(t)}{C}\right] B(t)-\mu_{B} B(t)-\alpha B(t) M(t) \\
\frac{d P(t)}{d t} & =k_{P}-\mu_{P} P(t)-\beta B(t) P(t) \\
\frac{d M(t)}{d t} & =k_{M}-\left(\mu_{M}+\overline{\mu_{B}}\right) M(t)+\gamma\left[P_{0}-P(t)\right]
\end{aligned}\right.
$$

Determina-se os pontos de equilíbrio e análise de estabilidade.

O ponto de equilíbrio trivial $Q_{1}^{0}$ do sistema (2.2) tem as coordenadas dadas por

$$
\left\{\begin{array}{l}
B=0 \\
P=P_{0}=\frac{k_{P}}{\mu_{P}} \\
M=\frac{k_{M}}{\mu_{M}+\overline{\mu_{B}}}
\end{array}\right.
$$

Esse equilíbrio corresponde, em caso de infecção, ao retorno do sistema imunológico ao estado basal, após debelar a infecção.

A estabilidade local de $Q_{1}^{0}$ é determinada pelos autovalores associados à matriz Jacobiana (linearização do sistema dinâmico) $J$,

$$
J=\left[\begin{array}{ccc}
\left(k_{B}-\mu_{B}\right)-\frac{2 B}{C}-\alpha M & 0 & -\alpha B \\
-\beta P & -\mu_{P}-\beta P & 0 \\
0 & -\gamma & -\left(\mu_{M}+\overline{\mu_{B}}\right)
\end{array}\right]
$$

calculada no ponto de equilíbrio com coordenadas dadas por (2.3). Os autovalores associados são $\lambda_{1}=-\frac{\left(\alpha-\alpha_{0}\right) k_{M}}{\mu_{M}+\overline{\mu_{B}}}, \lambda_{2}=-\mu_{P}$ e $\lambda_{3}=-\left(\mu_{M}+\overline{\mu_{B}}\right)$. O ponto de equilíbrio é localmente e assintoticamente estável se $\alpha>\alpha_{0}$. Portanto, $Q_{1}^{0}$ é um nódulo atrator para taxa de mortalidade suficientemente grande das bactérias causada pelos macrófagos. O sistema imunológico retorna ao estado basal após debelar infecção e se coloca em prontidão contra outras infecções.

O ponto de equilíbrio não trivial $Q_{1}^{*}$ tem as coordenadas dadas por

$$
\left\{\begin{array}{l}
P=\frac{k_{P}}{\mu_{P}+\beta B} \\
M=\frac{k_{M}+\gamma\left(\frac{k_{P}}{\mu_{P}}-\frac{k_{P}}{\mu_{P}+\beta B}\right)}{\mu_{M}+\mu_{B}},
\end{array}\right.
$$

onde o valor de $B$ é dado pelas soluções positivas da equação de segundo grau

$$
A_{2} B^{2}+A_{1} B+A_{0}=0,
$$

com os coeficientes dados por

$$
\left\{\begin{array}{l}
A_{2}=\left(\mu_{M}+\overline{\mu_{B}}\right) \beta k_{B} \\
A_{1}=k_{B} \mu_{P}\left(\mu_{M}+\overline{\mu_{B}}\right)+\beta C \alpha_{0}\left(k_{M}+\frac{\gamma k_{P}}{\mu_{P}}\right)\left[\frac{\alpha}{\alpha_{0}}-\frac{k_{M}}{k_{M}+\frac{\gamma k_{P}}{\mu_{P}}}\right] \\
A_{0}=C k_{M} \mu_{P} \alpha_{0}\left(\frac{\alpha}{\alpha_{0}}-1\right),
\end{array}\right.
$$


e o valor limiar de destruição de bactérias por macrófagos $\alpha_{0}$ é dado por

$$
\alpha_{0}=\frac{\left(\mu_{M}+\overline{\mu_{B}}\right)\left(k_{B}-\mu_{B}\right)}{k_{M}} .
$$

A existência de equilíbrio não trivial é estudada pela Regra de Descartes [7], com $A_{2}>0$ :

(i) Para $0<\alpha<\alpha_{0}$, como se tem $A_{0}<0$, então o polinômio (2.4) tem uma única solução. Assim, $Q_{1}^{*}$ é único. Quando a capacidade fagocitária de macrófagos for pequena, então a bactéria pode persistir no local de infecção.

(ii) Para $\alpha>\alpha_{0}$, tem-se duas possibilidades:

(ii.a) Se $k_{B}<\mu_{B}$, caso em que $\alpha_{0}<0$, tem-se $A_{0}>0$ e $A_{1}>0$, então o polinômio (2.4) não tem nenhuma solução positiva. Assim, $Q_{1}^{0}$ é o único ponto de equilíbrio. Nesta situação, a infecção é sempre debelada pelo sistema imunológico.

(ii.b) Se $k_{B}>\mu_{B}$, caso em que $\alpha_{0}>0$, tem-se $A_{0}>0$ e $A_{1}>0$, então o polinômio (2.4) não tem nenhuma solução.

A análise de estabilidade de $Q_{1}^{*}$ é feita numericamente.

O ponto de equilíbrio trivial $Q_{1}^{0}$ é localmente e assintoticamente estável para $\alpha>$ $\alpha_{0}$. Por sua vez, o único ponto de equilíbrio não trivial $Q_{1}^{*}$ só existe para $0<\alpha<\alpha_{0}$. Isto significa que, independentemente da inoculação inicial de bactérias, ou o sistema imunológico debela a infecção, ou ele escapa da reação inflamatória localizada. E o parasita pode cair na corrente sagüínea, pois a produção dos macrófagos ocorre a uma taxa constante, independentemente da concentração de antígenos. Nesse modelo ocorre uma bifurcação em $\alpha=\alpha_{0}$.

As simulações do sistema (2.2) mostram que, se a taxa de destruição das bactérias causada pelos macrófagos $\alpha$ for suficientemente grande, o sistema imunológico consegue debelar a infecção independentemente da quantidade inicial de bactérias inoculadas. No entanto, se essa taxa não for suficientemente grande, o organismo sofrerá uma infecção generalizada, devido à incapacidade do sistema imunológico em repelir o invasor.

\subsection{Macrófagos Atraídos pelas Células Mortas e Macrófagos}

Esse sub-modelo assume que novos macrófagos são atraídos por interleucinas, que são produzidas a uma taxa constante per-capita $\gamma$, sendo a produção total dada por $\gamma M$. O sistema dinâmico (2.1) fica

$$
\left\{\begin{array}{l}
\frac{d B(t)}{d t}=k_{B}\left[1-\frac{B(t)}{C}\right] B(t)-\mu_{B} B(t)-\alpha B(t) M(t) \\
\frac{d P(t)}{d t}=k_{P}-\mu_{P} P(t)-\beta B(p) P(t) \\
\frac{d M(t)}{d t}=k_{M}-\left(\mu_{M}+\overline{\mu_{B}}\right) M(t)+\gamma\left[P_{0}-P(t)\right] M(t) .
\end{array}\right.
$$


Determina-se os pontos de equilíbrio e análise de estabilidade.

O ponto de equilíbrio trivial $Q_{2}^{0}$ do sistema (2.6) tem as mesmas coordenadas dadas na equação (2.3), ou seja, do ponto $Q_{1}^{0}$. A estabilidade local de $Q_{2}^{0}$ é determinada pelos autovalores associados à matriz Jacobiana (linearização do sistema dinâmico) $J$,

$$
J=\left[\begin{array}{ccc}
\left(k_{B}-\mu_{B}\right)-\frac{2 B}{C}-\alpha M & 0 & -\alpha B \\
-\beta P & -\mu_{P}-\beta P & 0 \\
0 & -\gamma M & -\left(\mu_{M}+\overline{\mu_{B}}\right)
\end{array}\right]
$$

calculada no ponto de equilíbrio com coordenadas dadas por (2.3). Os autovalores correspondentes à matriz são dados pelas raízes de polinômio de terceiro grau

$$
\lambda^{3}+H_{2} \lambda^{2}+H_{1} \lambda+H_{0}=0
$$

onde os coeficientes são dados por

$$
\left\{\begin{array}{l}
H_{2}=\left[-\left(k_{B}-\mu_{B}\right)+\frac{\alpha K_{M}}{\mu_{M}+\overline{\mu_{B}}}+\left(\mu_{B}+\overline{\mu_{B}}\right)+\mu_{P}\right] \\
H_{1}=\left[-\left(k_{B}-\mu_{B}\right)+\frac{\alpha K_{M}}{\mu_{M}+\overline{\mu_{B}}}\right]\left[\mu_{P}+\left(\mu_{M}+\overline{\mu_{B}}\right)\right]+\mu_{P}\left(\mu_{M}+\overline{\mu_{B}}\right) \\
H_{0}=\left(\frac{\alpha}{\alpha_{0}}-1\right)\left(k_{B}-\mu_{B}\right) \mu_{P}\left(\mu_{M}+\overline{\mu_{B}}\right),
\end{array}\right.
$$

com o valor limiar de destruição de bactérias por macrófagos $\alpha_{0}$ sendo dado por $(2.5)$.

Segundo os critérios de Routh-Hurwitz, para que o polinômio de ordem 3, equação (2.7), tenha todas as raízes com parte real negativa, devemos ter: $H_{2}>0, H_{0}>0$ e $H_{2} H_{1}>H_{0}$ [3]. Analisa-se apenas o último termo $H_{0}$, conforme a conjectura que resume os critérios de estabilidade de Routh-Hurwitz feita em [6]. $H_{0}$ é menor do que zero para $\alpha<\alpha_{0}$; ou, para $k_{B}<\mu_{B}$, independente de valor de $\alpha$. Assim o equilíbrio trivial é localmente e assintoticamente estável apenas para $\alpha>\alpha_{0}$ e $k_{B}>\mu_{B}$. Note que $\alpha>\alpha_{0}$ e $k_{B}>\mu_{B}$ são as condições para não existir ponto de equilíbrio não trivial $Q_{1}^{*}$ do sub-modelo anterior.

O ponto de equilíbrio não trivial $Q_{2}^{*}$ tem as coordenadas dadas por

$$
\left\{\begin{array}{l}
B=\frac{k_{P}-\mu_{P} P}{\beta P} \\
M=\frac{k_{M}}{\left(\mu_{M}+\overline{\mu_{B}}\right)-\gamma\left(P_{0}-P\right)}
\end{array}\right.
$$

onde o valor de $P$ é dada pelas soluções positivas da equação de segundo grau

$$
A_{2} P^{2}+A_{1} P+A_{0}=0
$$

com os coeficientes dados por

$$
\left\{\begin{array}{l}
A_{2}=\gamma\left[\beta C\left(k_{B}-\mu_{B}\right)+k_{B} k_{P}\right] \\
A_{1}=\left(\mu_{M}+\overline{\mu_{B}}\right)\left[\beta C\left(k_{B}-\mu_{B}\right)+k_{B} k_{P}\right]-\beta \gamma C \frac{k_{P}}{\mu_{P}}\left(k_{B}-\mu_{B}\right)-\alpha\left(\beta C k_{M}+k_{B} k_{P}\right) \\
A_{0}=k_{B} k_{P}\left[\gamma \frac{k_{P}}{\mu_{P}}-\left(\mu_{M}+\overline{\mu_{B}}\right)\right] .
\end{array}\right.
$$


Para estudar a existência de equilíbrio não trivial do polinômio (2.8), faz-se a seguinte substituição

$$
\psi=P_{0}-P
$$

que corresponde à variação na população das células do tecido destruídas em função das toxinas liberadas pelas bactérias. Com essa mudança, as coordenadas do ponto de equilíbrio não trivial $Q_{2}^{*}$ são dadas por

$$
\left\{\begin{array}{l}
B=\frac{k_{P}-\mu_{P} P_{0}+\mu_{P} \psi}{\beta P_{0}-\beta \psi} \\
M=\frac{k_{M}}{\left(\mu_{M}+\overline{\mu_{B}}\right)-\gamma \psi},
\end{array}\right.
$$

mais a equação para $\psi$ dada por

$$
I_{2} \psi^{2}+I_{1} \psi+I_{0}=0
$$

onde os coeficientes são dados por

$$
\left\{\begin{array}{l}
I_{2}=\gamma\left[\beta C\left(k_{B}-\mu_{B}\right)+k_{B} k_{P}\right] \\
I_{1}=\beta C\left[\left(k_{B}-\mu_{B}\right)\left(\gamma P_{0}+\mu_{M}+\overline{\mu_{B}}\right)\left(\frac{\alpha}{\alpha_{1}}-1\right)-k_{B} \mu_{P}\left(\mu_{M}+\overline{\mu_{B}}\right)\right] \\
I_{0}=\beta C P_{0}\left(\mu_{M}+\overline{\mu_{B}}\right)\left(k_{B}-\mu_{B}\right)\left(1-\frac{\alpha}{\alpha_{0}}\right)
\end{array}\right.
$$

com o valor limiar de destruição de bactérias por macrófagos $\alpha_{0}$ sendo dado por (2.5), e o parâmetro $\alpha_{1}$ é dado por

$$
\alpha_{1}=\frac{\left(k_{B}-\mu_{B}\right)\left(\gamma P_{0}+\mu_{M}+\overline{\mu_{B}}\right)}{k_{M}} .
$$

A existência de equilíbrio não trivial é estudada pela Regra de Descartes [7], em que, para $k_{B}>\mu_{B}$, tem-se $\alpha_{0}<\alpha_{1}$ :

(i) Para $\alpha<\alpha_{0}$, tem-se que $I_{0}>0, I_{1}<0$ e $I_{2}>0$; e o discriminante do polinômio $(2.9)$,

$$
\begin{aligned}
\Delta= & {\left[\beta C\left(k_{B}-\mu_{B}\right)\left(\gamma P_{0}+\mu_{M}+\overline{\mu_{B}}\right)\left(\frac{\alpha}{\alpha_{1}}-1\right)-k_{B} \mu_{P}\left(\mu_{B}+\overline{\mu_{B}}\right)\right]^{2} } \\
& -4 \gamma\left[\beta C\left(k_{B}-\mu_{B}\right)+k_{B} k_{P}\right]\left[\beta C P_{0}\left(\mu_{M}+\overline{\mu_{B}}\right)\left(k_{B}-\mu_{B}\right)\left(1-\frac{\alpha}{\alpha_{0}}\right)\right],
\end{aligned}
$$

é tal que $\Delta>0$. Logo, o polinômio (2.9) tem duas raízes reais positivas.

(ii) Para $\alpha>\alpha_{0}$, tem-se $I_{0}<0$ e, independentemente do sinal do $I_{1}$, o polinômio (2.9) tem-se apenas uma única raiz real positiva.

A Figura 1 mostra o diagrama de soluções do polinômio (2.9). Na região em que $\alpha<\alpha_{0}$, tem-se $I_{0}>0$; em $\alpha=\alpha_{0}$, tem-se $I_{0}=0$; e em $\alpha>\alpha_{0}$, tem-se $I_{0}<0$. O ramo positivo da solução de polinômio (2.9) cresce a partir do valor $\psi=P_{0}$ em $\alpha=0$. O outro ramo, porém, decresce a partir do valor $\psi=P_{0}$ em $\alpha=0$, assume $\psi=0$ em $\alpha=\alpha_{0}$, e, posteriormente, assume valores negativos. Note que $\psi=P_{0}-P$, logo, deve-se ter $0 \leq \psi \leq P_{0}$ para ser biologicamente viável. 


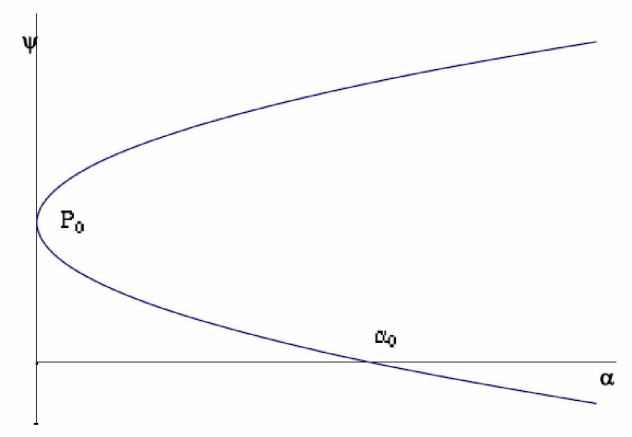

Figura 1: Solução de polinômio $(2.9), \psi$, com respeito ao valor da taxa de destruição de bactérias por macrófagos $\alpha$.

Assim, o ramo monotonicamente crescente não é biologicamente viável; apenas o ramo decrescente deve ser considerado.

A análise de estabilidade de $Q_{2}^{*}$ é feita numericamente.

Da mesma forma que o modelo anterior, o ponto de equilíbrio trivial $Q_{2}^{0}$ é localmente e assintoticamente estável para $\alpha>\alpha_{0}$ e $k_{B}>\mu_{B}$, e instável em outros casos. Por sua vez, o ponto de equilíbrio não trivial $Q_{2}^{*}$ positivo é biologicamente inviável para $\alpha>\alpha_{0}$ e $k_{B}>\mu_{B}$, e a solução decrescente assume valores negativos a partir desse valor. Assim, conjectura-se que o ponto de equilíbrio $Q_{2}^{*}$ biologicamente viável, dado pela raiz menor do polinômio (2.9), é localmente e assintoticamente estável para $\alpha<\alpha_{0}$ ou $\alpha>\alpha_{0}$ e $k_{B}<\mu_{B}$.

A Figura 1 mostra que, para qualquer ponto da região $\alpha<\alpha_{0}$, as trajetórias do sistema (2.6) tendem para o ramo biologicamente viável (ponto de equilíbrio não trivial), exceto quando não há bactérias, que é o ponto de equilíbrio trivial. Esse ponto é instável, e qualquer perturbação leva para equilíbrio não trivial. Para qualquer ponto da região $\alpha>\alpha_{0}$, as trajetórias tendem para o ponto de equilíbrio trivial, que é estável. Outro ponto de equilíbrio é instável, pois não tem sentido biológico.

\section{Conclusão}

Na realidade, as bactérias extracelulares estreptococos e estafilococos apresentam características distintas. Os estafilococos que invadem os tecidos liberam toxinas celulares extremamente letais. Enquanto os estreptococos não causam intensa destruição local. No entanto, observa-se que os estreptococos têm uma tendência muito maior do que os estafilococos a propagar-se por todo o organismo e causar morte, embora os estafilococos sejam muito mais destrutivos para os tecidos [5].

A explicação para este fenômeno reside na capacidade dessas bactérias em provocar reações imunes. Os estafilococos, ao destruírem mais células teciduais, como conseqüência, o processo de inflamação desenvolve-se rapdamente - na realidade, 
muito mais rapidamente do que os próprios estafilococos podem multiplicar-se e propagar-se. Por conseguinte, a infecção estafilocócica é tipicamente enclausurada com muita rapidez.

Por outro lado, como os estreptococos destroem menos células teciduais por serem menos patogênicos, assim, o processo de enclausuramento desenvolve-se lentamente, enquando os estreptococos reproduzem-se e migram. Conseqüentemente, têm mais chance de passar pela camada intersticial, onde ocorre reação inflamatória local. Ao se evadir de SI inato e se propagar para os outros órgãos através da corrente sangüínea, essas bactérias causam danos graves no corpo do hospedeiro.

O modelo simples que propomos descreve esse fenômeno. Suponhamos um sistema imunológico que cumpra suas funções, ou seja, $\alpha>\alpha_{0}$. Para um indivíduo sadio e imuno-competente, as bactérias que conseguem invadir a barreira física, em geral, são eliminadas. Quanto maior for a capacidade de destruição das células pelas toxinas liberadas pelas bactéria na sua multiplicação, mais vigorosa será a resposta imunológica, diminuindo a concentração de bactérias no local de inflamação (quantificado pelo parâmetro $\beta$ ). Porém, quando as bactérias atuam 'silenciosamente', em que toxinas são menos citotóxicas, ocorre a penetração de bactérias ao organismo humano. Isso se deve a pouca sinalização para atrair macrófagos ao local de invasão. Os estreptococos causam relativamente pouca destruição de células teciduais, o que induz a pouca migração dos macrófagos tendo, como resultado, uma baixa taxa per capita de mortalidade das bactérias pelos macrófagos $(\alpha M)$. Os estafilococos, contrariamente, provocam grande redução na população das células teciduais, e a tendência deles é desaparecer em função da alta mortalidade causada pelos macrófagos. Por isso, mesmo que os estafilococos sejam mais letais ao ser humano, quem causa maior incidência de morte são os estreptococos, menos patogênicos.

Se não houvesse resposta imunológica, as bactérias que tiverem elevada taxa de replicação $\left(k_{B}>\mu_{B}\right)$ perpetuariam, enquanto de baixa reprodução $\left(k_{B}<\mu_{B}\right)$, extinguiriam. Mas, quando o sistema imunológico responde potencializado pela produção de toxinas quando as bactérias se multiplicam, as que causam maiores danos às células teciduais são controladas pelo sistema imunológico, enquanto as bactérias menos letais às células têm possibilidades de escapar da contenção de sistema imunológico e, dependendo de estado imunológico do indivíduo, resultar em infecção generalizada.

Todos os resultados apresentados são válidos para valores de $\beta$ pequenos. Em um futuro trabalho será mostrada com detalhes a influência da capacidade destrutiva das toxinas $(\beta)$ na indução de uma resposta imunológica.

\footnotetext{
Abstract. When bacteria overcome physical barriers (skin or mucus of digestive and intestinal tracts), they induce local inflammatory reaction due to the action of innate immune system. We develop a mathematical model to describe the bacterial infection taking into account the pathogenicity. The results are applied to assess the invasion of two species of bacteria (staphylococcus and streptococcus) through the human skin. The model shows that the pathogenicity of bacteria plays a major role in the evasion of parasite to the innate immune response.
} 


\section{Referências}

[1] S. Barrozo, H. Yang, C.H. Dezotti, "Uma Abordagem Matemática em Imunologia", Matemática Aplicada à Fisiologia, pp. 93-117, SBMAC, São Carlos, 2003.

[2] M.C.C. Cunha, "Métodos Numéricos", 2ed., Editora Unicamp, 2000.

[3] L. Edelstein-Keshet, "Mathematical Models in Biology', Random House, New York, 1987.

[4] Z. Feng, C. Castilho-Chavez, C.A.F. Capurso, A Model for Tuberculosis with Exogenous Reinfection, Theorical Population Biology, 57 (2000), 235-247.

[5] A.C. Guyton, "Tratado de Fisiologia Médica", Ed. Guanabara Koogan, RJ, 1992.

[6] M.B.F. Leite, R.C. Bassanezi, H.M. Yang, The Basic Reproduction Ratio For a Model of Directly Transmitted Infections Considering The Virus Charge and The Immunological Response, IMA. J. Math. Appl. Med. Biol., 17, No. 1 (2000), $15-31$.

[7] J.D. Murray, "Mathematical Biology I: An Introduction", Ed. Springer, 1993.

[8] I. Robitt, A. Robson, "Imunologia Básica", Ed. Guanabara Koogan, RJ, 1992.

[9] H.M. Yang, "Epidemiologia Matemática - Estudo dos Efeitos da Vacinação em Doenças de Transmissão Direta", EDUCAMP e FAPESP, Campinas, 2001. 